\title{
Evolving Connectionist Systems Based Role Allocation of Robots for Soccer Playing
}

\author{
L. Huang, Q. Song and N. Kasabov
}

\begin{abstract}
For a group of robots (multi-agents) to complete a task, it is important for each of them to play a certain role changing with the environment of the task. One typical example is robotic soccer in which a team of mobile robots perform soccer playing behaviors. Traditionally, a robot's role is determined by a closed-form function of a robot's postures relative to the target which usually cannot accurately describe real situations. In this paper, the robot role allocation problem is converted to the one of pattern classification. Evolving classification function (ECF), a special evolving connectionist systems (ECOS), is used to identify the suitable role of a robot from the data collected from the robot system in real time. The software and hardware platforms are established for data collection, learning and verification for this approach. The effectiveness of the approach are verified by the experimental studies.
\end{abstract}

\section{INTRODUCTION}

Proper role allocation is essential for a group of robots to perform a highly intelligent task such as soccer playing. The environments of these tasks are dynamic, so the role allocation must also be performed dynamically [1]-[5]. In the robotic soccer, the roles of the robots are classified as attacker, defender and goal keeper, or, for a team of more than three robots, active, strategic, supports and goal keeper [2]. The attacker(active) chases the ball with various maneuvers and aims to put it into the opponent goal area; the defender (strategic) provides a defense to push the ball and the opponent robot away from the home goal area; the support acts as a support for the attacker or defender depending on the overall situations of the soccer playing.

Various methods are proposed for robot role allocation. In one of the most popular approaches [1][2][5], the prefered pose of each role is determined by strategic positioning using attraction and repulsion (SPAR) method. The relative postures among the robots, the ball and the prefered poses such as the relative distance and angles etc. are then examined. A numerical indication (utility) of each robot to each role is then produced as a weighted sum of relative postures. The robot will be allocated the role if it has the highest utility for it. In [3], an auction mechanism for resource allocation [4] is applied in robot role allocation. In this approach, matching functions based on the attributes of

L. Huang is with School of Electrical and Electronic Engr., Singapore Polytechnic, Singapore 139651. loulin@sp.edu.sg

Q. Song and N. Kasabov are with Knowledge Engr. and Discovery Research Institute, Auckland Univ of Tech. Private Bag 92006, Auckland 1020, New Zealand. \{qsong, nkasabov\}@aut.ac.nz the robots are derived for matching the offer(s) of each trader (robot) to the role(s) available. These approaches try to quantify the relationship between the states of the robotic soccer system (coordinates, distance and angle of the robots and the ball) and the robots' roles with one or several closed-form function(s) (utility or matching function). The real situations of the soccer playing are sometimes too complex to be described by those closed-form functions, and as a result, the accuracy and the reliability of the role allocation they generated is compromised.

If the roles of the robots are viewed as patterns (classes) and the states of the robotic system are viewed as a sequence of data changing with the process of the soccer playing, the problem of the robot role allocation can be stated as: given a data representing the states of the robotic system, what kind of pattern (robots' roles) does it belong to? This is a typical pattern classification problem for which many powerful tools exist such as principal components analysis (PCA) [6][7], neural network (NN) [6][8], support vector machines (SVM) [6][9] and evolving connectionist systems (ECOS) [10][11], to name a few. Though all the methods mentioned above can be applied, the ECOS method is adopted for its unique evolving feature and its successful applications in various evolving processes.

The following is the outline of the paper. In Section II, the problem formulation is described. Section III is dedicated to the study of ECOS-based robot allocation, its procedure and some practical issues involved. Section IV describes the experimental platforms for data collection and learning; the experimental results are also presented. The conclusion of the work is given in Section V.

\section{PROBLEM FORMULATION}

Robotic soccer is a good platform for studying the various issus of control of a group of robots (multiagents in general) [12][13]. In this paper, the small league robotic soccer game (three robots a side) introduced by Federation of International Robot-soccer Association (FIRA) is used for studying the role selection among the robots. The layout of the robotic soccer system is schematically shown in Figure 1 [13]. The robots of dimension within $75 \mathrm{~mm} \times 75 \mathrm{~mm} \times 75 \mathrm{~mm}$ are free to move in a field with dimension $150 \mathrm{~mm} \times 130 \mathrm{~mm}$. The common goal of the robot team is to push the ball into the opponent goal net. The states of the robots 


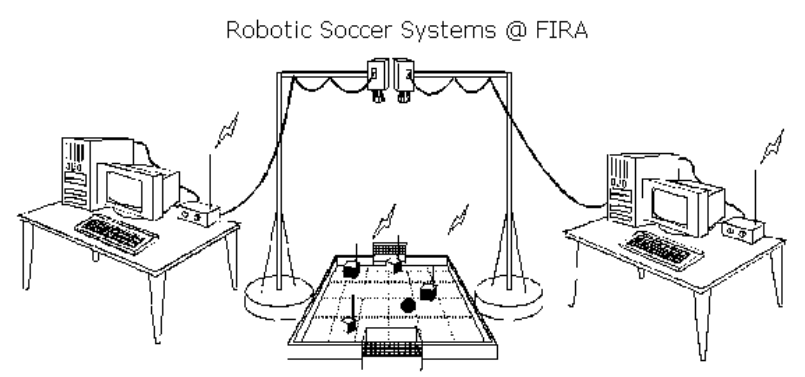

Fig. 1. Robotic Soccer System [13]

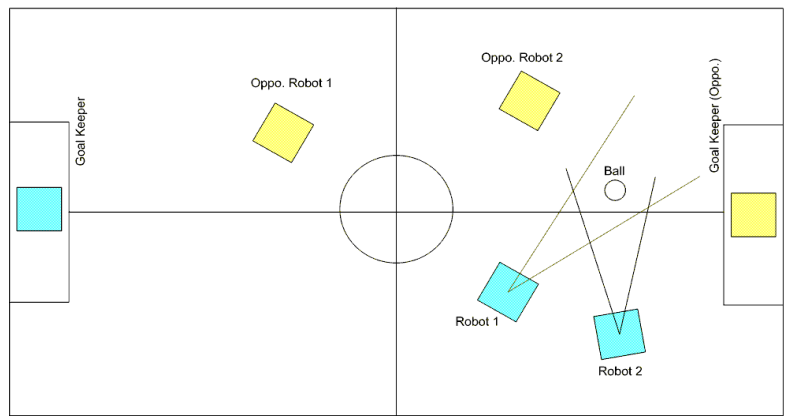

Fig. 2. One Scenario of Robotic Soccer

and the ball (target) are captured by the camera (with image processing systems) on the top of the field, and sent to the computer for the processing. The motion commands generated by the computer are sent to the robots through wireless communications between the computer and the robots.

To generate an effective defending or attacking formation by the robots, the roles (attacker, defender and goal keeper) are first assigned to the robots according to the the states of the robots and the ball. Figure 2 shows a scenario when two home robots are near the opponent goal area. Obviously the robot in the best attacking posture ( the position and the angle of the robot) should be assigned as an attacker. Though to quantify the best attacking posture is not an easy task, its dependence on the states of the robots and the ball is out of question. For Robot 1, its position is denoted by Cartesian coordinates $p_{1}=\left[\begin{array}{ll}x_{1} & y_{1}\end{array}\right]^{T}$ and its angle is denoted by $\theta_{1}$. Similarly, the position of Robot 2 are denoted by $p_{2}=\left[\begin{array}{ll}x_{2} & y_{2}\end{array}\right]^{T}$ and $\theta_{2}$ respectively. The ball position is represented by $p_{b}=\left[\begin{array}{ll}x_{b} & y_{b}\end{array}\right]^{T}$.

Combining the postures of the robots and the ball, the following vector at the moment $t$ is defined:

$$
p(t)=\left[p_{b}^{T}(t) p_{1}^{T}(t) \theta_{1}(t) p_{2}^{T}(t) \theta_{2}(t)\right]^{T}
$$

The argument $t$ is not shown when no ambiguity is caused by doing so in the following discussion.

The robot role selection problem now becomes, Given

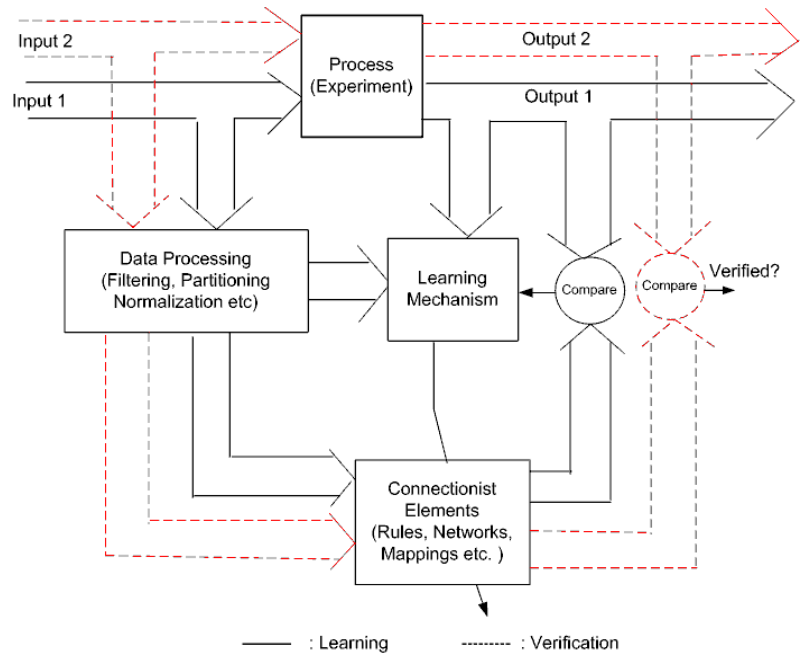

Fig. 3. A Simplified ECOS with Supervised Learning

$p(t)$, which robot, Robot 1 or Robot 2 should be attacker ? After the attacker is selected, the remaining robot should be defender. In the following section, the concept of evolving classification function is used to tackle this problem.

\section{Role Selection based on Evolving Classification Function (ECF)}

\section{A. Evolving Classification Function}

Evolving connectionist system (ECOS) is a connectionist architecture that facilitates modeling of an evolving process and knowledge discovery. It consists of a collection of networks that operate continuously in time and adapt their structures through continuous interaction with the environment and other systems [10]. How to adapt their structures is the task of the learning mechanism (supervised or unsupervised) in the system. ECOS is a very general concept which encompasses memory based, statistical or symbolic knowledge extracted from the incoming data to the system. Figure 3 is the block diagram of a much simplified ECOS with supervised learning. In the figure, the data Input 1 and Output 1 are for the learning, and the data Input 2 and Output 2 are for verification. The solid line shows the learning process and the dashed line shows the verification process. The structure of this simplified ECOS is similar to those of common supervised learning systems, but it is unique in its learning mechanism which is able to cater for an evolving process. For example, the structure as well the parameters of the connectionist elements (neural network, rules etc) are subject to change.

Evolving classification function is a special ECOS used for pattern classification [10][11]. It generates rule nodes in an $N$-dimensional input space and associate 


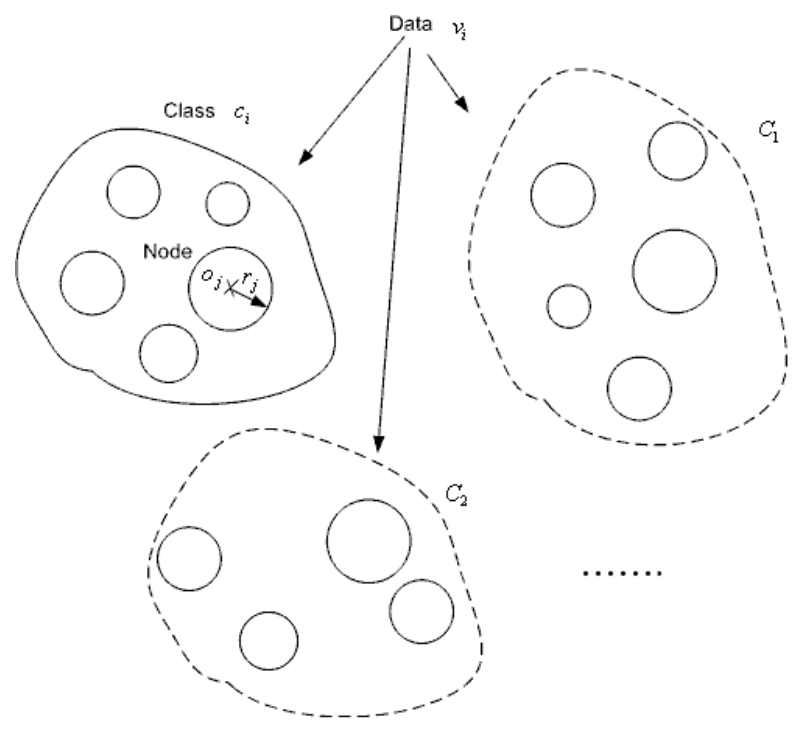

Fig. 4. Data Classification

them with classes. Each rule node is defined by its center and radius (influence field) in addition to the class it belongs to. A learning mechanism is designed in such a way that the new nodes can be generated, the current nodes can be merged or deleted with the incoming data reflecting.

The following notations are used to describe an ECF: $C: \quad$ the class set;

$v_{i}: \quad$ the $i$ th data vector;

$c_{i} \in C: \quad$ the class associated with $v_{i}$;

$o_{j}: \quad$ the center of $j$ th node;

$r_{j}: \quad$ the radius of $j$ th node;

$N_{j} \triangleq\left(o_{j}, r_{j}, c_{j}\right): \quad$ the $j$ th node of $c_{j} \in C$

$d_{\text {min }}$ : the minium radius of a node;

$d_{\max }: \quad$ the maximum radius of a node.

where $i$ and $j$ are whole number and their ranges are determined by the number of data and nodes. Figure 4 schematically shows the classification of a set of data.

There are learning phase and recall phase in the operation of ECF. In the learning phase, the following learning algorithm is executed in each iteration:

- Step 1: Initialize $i=0, j=0$ and $N_{0}=$ $\left(v_{0}, d_{\min }, c_{0}\right)$.

- Step 2: If all the data vectors are inputted, end the learning phase; otherwise, input vector $v_{i}$ and its class $c_{i}$; add 1 to $i$.

- Step 3: Add 1 to $j$ and go to Step 2; otherwise, calculate the distance between $v_{i}$ and the centers of all the created nodes: $d_{i j}=\left\|v_{i}-o_{j}\right\|(j=1 \ldots m$ where $m$ is the number of the nodes created);

- Step 4: If $d_{i j}>d_{\max }$ for ALL $j$, increase $j$ by 1 , create a new node $N_{j}=\left(v_{i}, d_{\min }, c_{i}\right)$ and go to Step 2; otherwise:

- Step 5: If $d_{i j} \leq r_{j}$ and $c_{i}=c_{j}$ for any one of $j$, do nothing and then go to Step 2; otherwise:

- Step 6: If $d_{i j} \leq r_{j}$ and $c_{i} \neq c_{j}$ for ALL $j$, the radius of the current node is adjusted such that $r_{j}=\max \left(d_{\min }, d_{i j}-d_{\min }\right)$ and then go to Step 2; otherwise:

- Step 7: If $d_{i j} \leq d_{\max }$ and $c_{i}=c_{j}, N_{j}$ is adjusted such that $r_{j}=d_{i j}$ unless $N_{j}$ doesn't cover other nodes; else add $j$ by 1 , set $N_{j}=\left(v_{i}, d_{\min }, c_{i}\right)$ and go to Step 2.

The final result of the learning phase is a list of (rule) nodes.

In the recall phase, a new data vector (different from the ones used for the learning) is examined against the established list of the nodes, and the class $\left(c_{i}\right)$ of the vector is identified. Here are the steps involved:

- Step 1: Input the vector $v_{i}$.

- Step 2: Calculate $d_{i j}=\left\|v_{i}-o_{j}\right\|$ for any nodes. If $d_{i j} \leq r_{j}$ and $c_{j}$ is unique, $c_{i}=c_{j}$ and go to Step 1 ; otherwise

- Step 3: If $d_{i j} \leq r_{j}$ and $c_{j}$ are not unique or $d_{i j}>r_{j}$ for ALL $j, c_{i}$ is the same as that of the node with the minimum $d_{i j}$ and then go to Step 1 .

Obviously, for the role selection in robotic soccer, $\mathrm{C}=\{$ Robot 1 is the attacker, Robot 2 is the attacker $\}$. The data $v_{i}$ for learning are derived from the processing on the raw data set $p$.

\section{B. Data Processing}

The vector $p$ is obtained from the image processing system in which various filtering techniques are used to eliminate noises. Even though, further processing on the vector $p$ is needed. We will transform $p$, which contains elements (position and angle) with different units, to another vector whose elements are homogeneous with each other. The data will also be partitioned according to the relative posture of the robot with respect to the ball.

From the vector $p$, the following variables describing the relative postures among the robots and the ball are derived: $d_{12}=\left\|p_{1}-p_{2}\right\|, \theta_{12}=\left\|\theta_{1}-\theta_{2}\right\|, d_{i b}=$ $\left\|p_{b}-p_{i}\right\|, \theta_{i b}=\angle\left(p_{b}-p_{i}\right), \gamma_{i b}=\left\|\theta_{b}-\theta_{i}\right\|$ and $d_{i g}=\|(L-$ $\left.x_{i}\right) \tan \left(\theta_{i b}\right)-W / 2 \|$, where $L=150 \mathrm{~cm}$ and $W=130 \mathrm{~cm}$ are the length and the width of the field respectively.

From the above variables, a new vector $p_{t}=\left[w_{1} *\right.$ $\left.p_{t 1} w_{2} * p_{t 2} \ldots w_{6} * p_{t 6}\right]^{T}$ is obtained:

$$
\begin{array}{ll}
p_{t 1}=\gamma_{1 b} /\left(\gamma_{1 b}+\gamma_{2 b}\right) & p_{t 2}=d_{1 b} /\left(d_{1 b}+d_{2 b}\right) \\
p_{t 3}=d_{1 g} /\left(d_{1 g}+d_{2 g}\right) & p_{t 4}=\gamma_{2 b} /\left(\gamma_{1 b}+\gamma_{2 b}\right) \\
p_{t 5}=d_{2 b} /\left(d_{1 b}+d_{2 b}\right) & p_{t 6}=d_{2 g} /\left(d_{1 g}+d_{2 g}\right)
\end{array}
$$

and $w_{i}(i=1,2 \ldots 6)$ are the weights for adjusting the contribution of each element to the role selection. By default, $w_{i}=1$. Note that $p_{t i}(i=1,2 \ldots 6)$ contains the important information about the robot postures in relation to the attacking ball such as the relative distance to the ball and angles to the ball and the 
opponent goal. All the elements of $p_{t}$ are normalized to be unit-free. After the above processing, $p_{t}$ can be used as a vector $v_{i}$ for the learning in ECF operation.

For better classification of data, the raw data $p$ are partitioned according to the position of the ball with respect to the robots such as

- Case 1: $x_{b}>x_{1}$ and $x_{b}>x_{2}$ (The ball is in front of all the robots);

- Case 2: $x_{2}<x_{b}<x_{1}$ or $x_{1}<x_{b}<x_{2}$ ( The ball is between the robots);

- Case 3: $x_{b}<x_{1}$ and $x_{b}<x_{2}$ (The ball is behind all the robots0.

where the relative position "in front", "between" and "behind" are in reference to the attacking direction. Another criteria for data partition are the distance between the robots and the ball such as

- Case $4: d_{1 b} \geq k_{f a r} d_{2 b}$ or $d_{2 b} \geq k_{f a r} d_{1 b}$ ( Big difference between the relative distances to the ball of two robots);

- Case 6: $d_{1 b}<k_{f a r} d_{2 b}$ and $d_{2 b} \leq k_{f a r} d_{1 b}$ (Normal difference between the relative distances to the ball of two robots).

where $k_{f a r}$ is a constant. If combining the above two sets of criteria, we can have six criteria for data partition. For each partition, the ECF operation is run and a list of nodes is generated separately. This also applies in the recall process.

\section{Experimental Platforms and Results}

The first challenge of applying ECF in the robotic soccer, a fast real time dynamic system, is the data collection. It is very tedious to place the robots/ball on the field and record theirs postures and corresponding roles manually. To make the data collection and learning more efficient and comprehensive, we develop a software package in which the robotic soccer process captured by the image processing system in real time is replayed on the computer screen. The user can select the roles of the robots interactively through a user friendly graphic user interface (GUI). The data sets for ECF learning are automatically generated and saved in a file.

Figure 5 shows a GUI for data collection. On the scree, one scene captured from the real robotic soccer game shown in Figure 6 is replayed. The robot is represented by a color square attached with its identity number ( 1 or 2 ). The line goes through the rectangle shows the direction of the robot. The ball is represented by a circle. By clicking the button "..Last $>>$ " or ">> Next..", the robotic soccer playing process can be played backward or forward. Examining the scene on the screen, we can select Robot 1 as the attacker by clicking the button "ONE", or Robot 2 as the attacker by clicking the button "TWO". A part of the data set for the case when the ball is in front of both robots is shown in Figure IV. The data processing, learning and recall

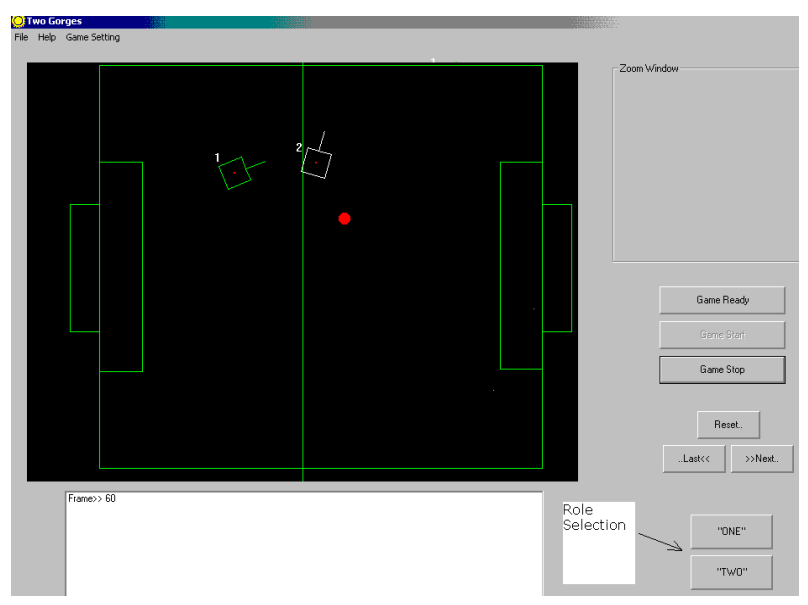

Fig. 5. Platform for Data Collection

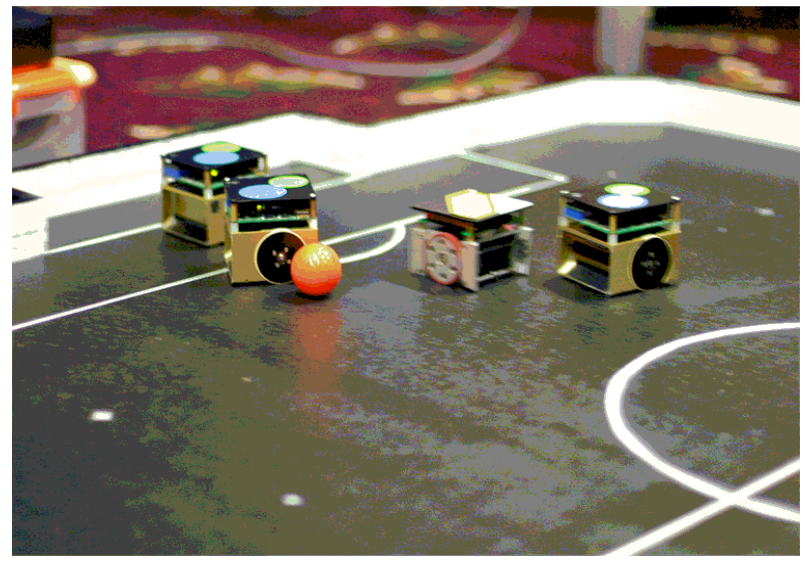

Fig. 6. Robots in Action in a Robotic Soccer Game

processes described in Section 3 are all integrated into a software package for the robotic soccer system, and one of the GUIs for these processes is shown in Figure 7. In one of the experiments, totally 122 data are collected for the case when the ball is in front of the robots. These data are divided into two sets, one with 82 data for learning and another with 40 data for verification. The parameters for learning are set as $d_{\text {min }}=0.01$, $d_{\text {max }}=0.15, w_{i}=1(i=1,2, \ldots 8), k_{f a r}=1.5$. After the learning process, 30 ECF rule nodes are generated, among which 10 nodes are shown in Figure ??. In the recall process for verification, the classes of 39 data (from 40 data) are identified correctly. The success rate is $97.5 \%$. The successful rates are also nvery consistent in other experiments.

The role selection approach presented above has been implemented in the robotic soccer teams - TWO GORGES and SPARIC from Singapore Polytechnic. The team TWO GORGES won Silver medal in the Singapore Robotic Games 2004 in May 2004 and the 


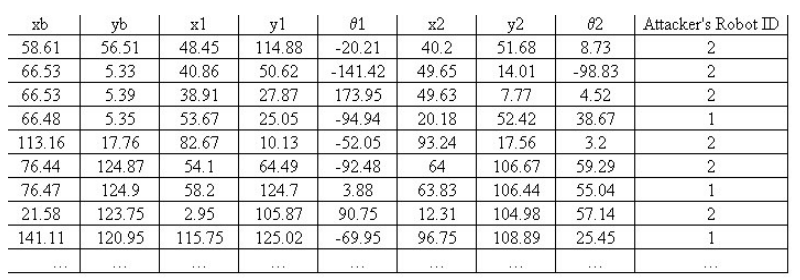

Table 1: Raw Data Collected (Partial)

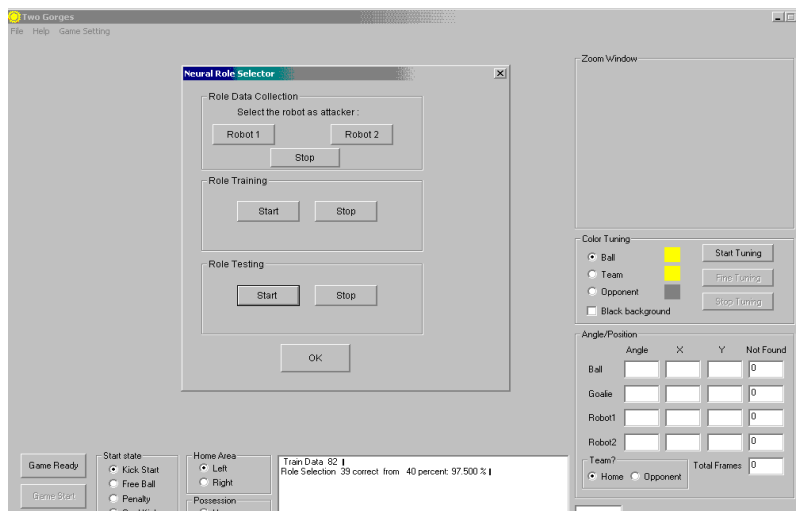

Fig. 7. GUI for ECF Learning and Verification

team SPARIC won the Gold medal in the large league and the Silver medal in the middle league in the 9th FIRA Robot World Cup held in Korea in Oct 2004.

\section{Conclusion}

This paper addresses the issue of robot role selection for soccer playing based on the concept of evolving connectionist system (ECOS). The role selection problem is converted into one of pattern classification and an evolving classification function, a special ECOS, is applied to solve it. The development of an integrated software platform for learning and game execution is described. The experimental study and results are presented to demonstrate the effectiveness of the approach.

\begin{tabular}{|c|c|c|c|c|c|c|c|}
\hline & \multicolumn{5}{|c|}{ Cortor } & Fadies & Das \\
\hline 0.000067 & 0.687735 & 0.07988 & 0.09093 & 0312265 & 092012 & 0.01 & 2 \\
\hline 0.011935 & 0.797166 & 0.530409 & 0.988065 & 0202834 & 0.469591 & 0.100786 & 2 \\
\hline 0.02896 & 0.548686 & 0.090924 & 0.971132 & 0.451114 & 0900076 & 0.01 & 1 \\
\hline 0.042639 & 0.373441 & 0.054242 & 0.957362 & 0626559 & 0945759 & 0.162074 & l \\
\hline 0.068601 & 0.462405 & 0.597472 & 0.931390 & 0537595 & 0.402528 & 0.01 & 1 \\
\hline 0.085874 & 0.598857 & 0.39181 & 0.914126 & 0.401143 & 060819 & 0.01 & 2 \\
\hline 0.094402 & 0.192386 & 0.07488 & 0.905598 & 0807614 & 092512 & \begin{tabular}{|l|l|}
0.17178 \\
\end{tabular} & 1 \\
\hline 0.135132 & 0.745151 & 0.092002 & 0.864868 & 0254849 & 0907908 & 0.01 & 2 \\
\hline 0.140514 & 0.467248 & 0.509745 & 0.859486 & 0532752 & 0.490255 & 0.01 & 1 \\
\hline 0.152978 & 0.401701 & 0.278058 & 0.847022 & 0598299 & 0.721942 & 0.01 & 1 \\
\hline
\end{tabular}

Table 2: ECF Nodes (Partial)

\section{References}

[1] P. Stone, M. Veloso, and P. Riley, CMUnited-98: Robocup-98 simulator world champion team, RoboCup-98:Robot SoccerWorld Cup II, M. Asada and H. Kitano, Eds. Berlin, Germany: Springer-Verlag, pp. 6176, 1999.

[2] T. Weigel, J.-S. Gutmann, M. Dietl, A. Kleiner and B. Nebel, "CS Freiburg: Coordinating Robots for Successful Soccer Playing," IEEE Trans. on Robotics and Auto., Vol 18, No 5, pp. 685-699, 2002.

[3] V. Frias-Martinez1, E. Sklar1, and S. Parsons, "Exploring Auction Mechanisms for Role Assignment in Teams of Autonomous Robots," Proceedings (CD-ROM) of ROBOCUP2004 Symposium, Instituto Superior Tcnico, Lisboa, Portugal, July 4-5, 2004.

[4] M. Esteva and J. Padget, "Auctions without Auctioneers: Distributed Auction Protocols," Agent-mediated Electronic Commerce II, LNAI 1788, pages 2028. Springer-Verlag, 2000.

[5] P. Stone and M. Veloso, "Task Decomposition, Dynamic Role Assignment, and Low-Bandwidth Communication for RealTime Strategic Teamwork", Artificial Intelligence, 110(2):241273, June 1999.

[6] R. O. Duda, P. E. Hart and D. G. Stork, Pattern Classification, John Wiley \$ Sons, Inc., 2001.

[7] S. Amari, A. Cichocki and H. Yang, "Blind Signal Separation and Extraction:neural and Information-theoretic Approach," in Unsupervised Adaptive Filtering, Vol 1 (ed. S. Haykin), John Wiley \& Sons, New York, pp. 63-138, 2000.

[8] S. Haykin, Neural Networks - a Comprehensive Foundation, Prentice Hall, Englewood Cliffs, NJ, 1994.

[9] V. Keceman, Learning and Soft Computing (Support Vector Machines, Neural Networks, and Fuzzy Systems), MIT Press, Cambridge, MA, 2001.

[10] N. Kasabov and Q. Song, GA-Optimization of Evolving Connectionist Systems for Classification with a Case Study from Bioinformatics, Proc. of ICONIP2002, Singapore, November, 2002.

[11] N. Kasabov, Evolving Connectionist Systems, Springer, 2002.

[12] M. Asada et.al, "An Overview of RoboCup-2002 Fukuoka/Busan", AI Magazine, 24(2), pp.21-40, Springer 2003.

[13] FIRA Official Web Site, "http://www.fira.net", visited on $26 / 11 / 2004$. 